\title{
Structural and electrochemical investigation of waste newspaper based electrodes for supercapacitor applications
}

\author{
T. AdinAVEen ${ }^{1 *}$, J. Judith ViJAYA $^{1}$, R. SivakumaR $^{1}$, L. John KenNedy ${ }^{2}$ \\ ${ }^{1}$ Catalysis and Nanomaterials Research Laboratory, Department of Chemistry, Loyola College, Chennai 600034 , India \\ ${ }^{2}$ Materials Division, School of Advanced Sciences, Vellore Institute of Technology (VIT) University, \\ Chennai Campus, Chennai 600 127, India
}

\begin{abstract}
There is a general consensus to develop renewable energy storage and conversion technologies to replace fossil fuel energy for sustainable development. Currently, the development of high performance energy storage and conversion devices is an important step on the road to alternative energy technologies. With a special focus on the upgradation of waste to valuable energy, this paper presents an effective synthetic method that utilizes waste newspapers as the precursor to prepare the activated carbon electrodes by the pyrolysis and chemical activation processes. The amorphous nature and surface morphology of the carbon samples were confirmed by XRD and SEM analysis, respectively. Activated waste newspaper carbon (AWNP) showed good electrochemical properties at $800{ }^{\circ} \mathrm{C}$ and its specific capacitance at a scan rate of $2 \mathrm{mV} / \mathrm{s}$ was found to be $380 \mathrm{~F} / \mathrm{g}$. It is important to mention that the source of the raw material is cost effective and suitable for green technology.
\end{abstract}

Keywords: waste newspaper; electrode; hierarchical porous carbon; supercapacitor

(C) Wroclaw University of Technology.

\section{Introduction}

Development of clean, green, renewable, and sustainable energy systems has gained much attention during the past decade due to the increase in the environmental pollution, and waste matter. It is mainly caused by the development of human activity that pose a continuously growing and serious problem. Since energy conversion and utilization are not necessarily concurrent events, efficient and durable energy storage is considered as important for the energy conversion from renewable sources [1]. Materials with purifying capability could be prepared from these wastes, and then they would be highly useful in overcoming both the pollution and waste problems simultaneously [2]. Paper is made up of cellulosic fiber sourced from plants. After consumption, it often makes its way to trash bins and thus comes to be termed as "waste paper". According to a recent report, about one-fifth of the contents of household waste bins consist of paper, of which half is newspaper

*E-mail: adinavee@gmail.com and magazines. This is equal to over $5 \mathrm{~kg}$ of wastepaper per family each week. Waste newspaper (WNP) is a printed paper, which has lost its useful value, because the information provided in it has become outdated. WNP mainly contains organic matter (mainly lignocellulose), as well as binding agents, printing inks, and auxiliary materials (plasticizers, fillers, surfactants, preservatives) [3-5].

At present, in 2014, the mixing ratio of waste paper to paper products and cardboard is $73.3 \%$, and hence if this mixing ratio continues until the year 2020, nearly $76,000,000$ tons of unutilized waste paper will be produced [6]. According to Indian Paper Manufacturers Association (IPMA), India is the fastest growing market for paper globally and it presents an exciting scenario; paper consumption is poised for a big leap forward in sync with the economic growth, and is estimated to touch 13.95 million tons by 2015 to 2016 . In India only about $10 \%$ waste paper is being currently recovered annually. Lack of segregation source results in waste paper getting contaminated 
and becoming unusable. Therefore, the development of applications for waste paper other than its utilization for manufacturing paper is demanded [5]. Recycle or reuse is the best current solution for reducing the disposal problem [7]. Compared to fossil fuels, renewable biomass resources have attracted a great deal of attention as alternative energy sources that protect the environment because they produce fewer greenhouse gas emissions [8].

Supercapacitors are a unique type of highpower electrochemical energy storage devices being developed for a wide range of uses, which includes consumer electronics, electrical utilities, medical electronics, transportation, and military defense systems. However, the energy and power densities, safety, and cycle life of currently available supercapacitors need to be modified significantly to satisfy the rapidly increasing performance demands for the aforementioned and many other applications. Therefore, the development of new electrodes and new electrolytes with superior properties is essential [9]. At present, activated carbon has been used as an electrode material in electrochemical double-layer capacitors (EDLCs), due to their well-developed manufacturing technologies, easy production in large quantities, relatively low cost and great cycle stability. AC is the oldest and most common type of porous carbons. The use of AC in Egypt was described as early as in 1550 BC [10]. Industrial production of ACs in U.S. started in 1913 [11]. Petroleum coke, pitch and coal are used to be the most common precursors for commercial AC production, but the decreasing ease of mining fossil fuels, increasing global energy demand, and increased awareness of the environmental impact of fossil fuel combustion has led to the AC production from sustainable and renewable resources, such as nutshells, wood, starch, sucrose, cellulose (WNP), corn grain, banana fiber, coffee grounds, sugar cane bagasse, and others.

To the best of our knowledge, only one report [12] exists on the preparation of activated carbon from waste newspaper (WNP) for electrical double layer capacitors (EDLCs) applications. In addition, no report is available on the activated carbon prepared from WNP using $\mathrm{H}_{3} \mathrm{PO}_{4}$ activation for the fabrication of supercapacitor electrodes. Thus, in this work, we made an attempt to assess the specific capacitance of the WNP activated carbons as electrode materials for EDLC applications. Hence, the study we conducted here is unique in this regard.

\section{Materials and methods}

\subsection{Preparation of porous carbons}

The porous carbon was prepared in the laboratory using waste newspaper (WNP) carbon as the precursor material by a two-stage process: precarbonization and chemical activation. In the precarbonization process, the WNP carbon was heated at $400{ }^{\circ} \mathrm{C}$ at a rate of $5{ }^{\circ} \mathrm{C} / \mathrm{min}$ for about $4 \mathrm{~h}$ and cooled down to room temperature at the same rate. It was labeled as precarbonized carbon (PCC). The PCC was subjected to chemical activation in order to create porous structure in the carbon matrix. In the chemical activation process, $50 \mathrm{~g}$ of the PCC was mixed with $250 \mathrm{~g}$ of aqueous solution containing $85 \% \mathrm{H}_{3} \mathrm{PO}_{4}$ by weight. The chemical activator and PCC were homogeneously mixed at $85{ }^{\circ} \mathrm{C}$ for $4 \mathrm{~h}$. After mixing, the PCC slurry was dried under vacuum at $110{ }^{\circ} \mathrm{C}$ for $24 \mathrm{~h}$. The resulting samples were then activated in a vertical cylindrical furnace at a flow rate of $5{ }^{\circ} \mathrm{C} / \mathrm{min}$. Then, it was labelled as activated waste newspaper (AWNP). This was followed by heating at three different temperatures of $600{ }^{\circ} \mathrm{C}, 700{ }^{\circ} \mathrm{C}$ and $800{ }^{\circ} \mathrm{C}$ at a heating rate of $5{ }^{\circ} \mathrm{C} / \mathrm{min}$ using a programmer and maintained at the constant temperature for $1 \mathrm{~h}$ before cooling. After cooling, the activated carbon was washed successively for several times with hot water until the $\mathrm{pH}$ became neutral and finally with cold water to remove the excess phosphorous compounds. The washed samples were dried at $110{ }^{\circ} \mathrm{C}$ to get the final product. The samples were labelled as AWNP 600, AWNP 700 and AWNP 800, respectively.

\subsection{X-ray diffraction studies}

$\mathrm{X}$-ray diffraction experiments were performed using a Phillips X'Pert diffractometer for $2 \theta$ values ranging from $10^{\circ}$ to $80^{\circ}$ using $\mathrm{CuK} \alpha$ 
radiation at a wavelength of $\lambda=1.540 \AA$. The other experimental conditions included $1 / 2^{\circ}$ divergence slits, a 5-s residence time at each step and intensity measurements in counts.

\subsection{FT-IR studies}

A Perkin Elmer infrared spectrometer was used for the investigation of surface functional groups of the porous carbon. The carbon samples were mixed with $\mathrm{KBr}$ of spectroscopic grade and shaped into pellets at a pressure of about $1 \mathrm{MPa}$. The pellets were about $10 \mathrm{~mm}$ in diameter and $1 \mathrm{~mm}$ in thickness. The samples were scanned in the spectral range of $4000 \mathrm{~cm}^{-1}$ to $400 \mathrm{~cm}^{-1}$.

\subsection{Surface morphological studies}

Surface morphology measurements were carried out on the samples using a Leo-JEOL scanning electron microscope. The carbon samples were coated with gold by a gold sputtering device for improving visibility of the surface morphology.

\subsection{Nitrogen isotherms}

$\mathrm{N}_{2}$ adsorption-desorption isotherms of the activated carbon composites were measured using an automatic adsorption instrument (Quantachrome Corp., Nova-1000 gas sorption analyzer) for determination of the surface area and the total pore volume.

\subsection{Electrical conductivity studies}

The DC electrical conductivity $(\sigma)$ was measured at room temperature by a two-probe method. $1 \mathrm{~g}$ of porous carbon dried at $110{ }^{\circ} \mathrm{C}$ over night was compressed in a hollow Pyrex glass cylinder with an inner diameter of $10 \mathrm{~mm}$ between two metal plungers at compression pressure ranging from $75.9 \mathrm{kPa}$ to $742 \mathrm{kPa}$. The top and the bottom of the plungers were made of copper. They were connected to a Keithly 614 electrometer to measure the electrical conductivity of the samples. The compression pressure throughout the measurements was maintained by placing the carbon loaded metal plungers (sample holder) inside a compression pressure hydraulic press. $3 \mathrm{~min}$ were allowed before every measurement of conductivity to avoid any transient effect of sample relaxation due to compression. The electrical conductivity was calculated using the following formula [13]:

$$
\sigma=\frac{L}{R A}
$$

where $\mathrm{L}$ is the distance between the two pistons $[\mathrm{cm}], \mathrm{R}$, the electrical resistance $[\Omega]$ and $\mathrm{A}$, the area of the piston surface $\left[\mathrm{cm}^{2}\right]$.

\subsection{Electrochemical measurements}

The electrodes were prepared by mixing 95 wt.\% activated carbon and 5 wt.\% polyvinylidenefluoride in ethanol to form a paste and then the slurry was coated onto a stainless steel plate, which served as a current collector. The coated electrode was dried at $70{ }^{\circ} \mathrm{C}$ in a drying oven for $2 \mathrm{~h}$ to remove the organic solvents remaining in the micropores of the electrode, and then the electrode weight was measured to determine the amount of carbon coated on the stainless steel sheet. A typical mass of electrode material was found to be $0.5 \mathrm{mg}$. All the measurements were done at room temperature. Cyclic voltammogram, galvanostatic charge, discharge, and electrochemical impedance spectroscopy measurements were performed using Autolab model PGSTAT $302 \mathrm{~N}$ with three electrode system. The carbon coated on the stainless steel sheet, $\mathrm{Ag} / \mathrm{AgCl}$ and platinum wire were used as the working, reference and counter electrode, respectively. Cyclic voltammetry (CV) studies of these electrodes were performed in $1 \mathrm{M} \mathrm{H}_{2} \mathrm{SO}_{4}$ electrolyte. Galvanostatic charge/discharge measurements were conducted from $-1.0 \mathrm{~V}$ to $0 \mathrm{~V}$ at different constant current densities from $15 \mathrm{~A} / \mathrm{g}$ to $5 \mathrm{~A} / \mathrm{g}$. EIS measurements were recorded in the frequency range of $10^{5} \mathrm{~Hz}$ to $10^{-2} \mathrm{~Hz}$ at a constant direct current bias potential of $0.2 \mathrm{~V}$ with the amplitude of $5 \mathrm{mV}$.

\section{Results and discussion}

\subsection{X-ray diffraction studies}

$\mathrm{X}$-ray diffraction technique provided the important information about the pore structure of the WNP carbon used in this study. The internal structure of the carbon was considered 
to be the most important factor [13]. Fig. 1 shows the X-ray diffraction pattern of the precarbonized and activated carbon samples of WNP carbon. The strong $\mathrm{C}\left(\begin{array}{lll}0 & 0 & 2\end{array}\right)$ peak shows the graphitic carbon at $2 \theta(\mathrm{CuK} \alpha)$ of $23.1^{\circ}$, due to the hexagonal graphite type structure, demonstrating the turbostratic carbon structure with randomly oriented graphitic carbon layers [14]. Moreover, the peak around $44.3^{\circ}$ is due to the formation of a higher degree of intralayer condensation, which should greatly improve the electrical conductivity [15]. In addition, this may be assigned to the (10) bidimensional planes [16]. The existence of $\mathrm{h} \mathrm{k}\left(\begin{array}{ll}1 & 0\end{array}\right)$ lines may be due to the turbostatic or convoluted stacking of hexagonal layers of carbon resulting in the disordered structure and lower crystallinity $[17,18]$. The intensity increase at the low-angle scattering peak indicates a high density of micropores. Hence, the results show that all the carbon materials have a certain degree of graphitic structure, and the decrease in peak intensity indicates the amorphous state of the material.

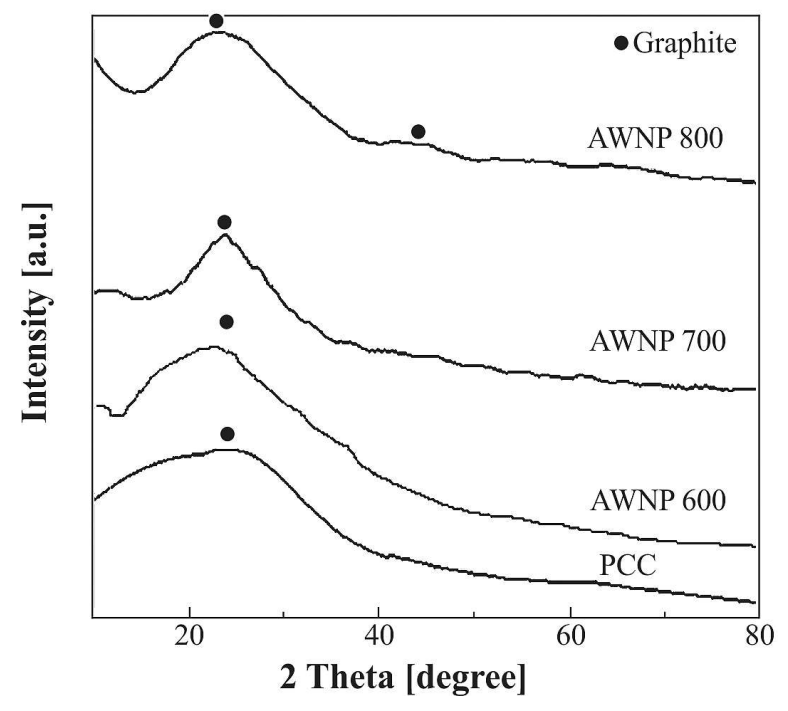

Fig. 1. X-ray diffraction patterns of (a) PCC, (b) AWNP 600, (c) AWNP 700, (d) AWNP 800 carbons

\subsection{Fourier transform infrared (FT-IR) spectroscopy studies}

Fig. 2 shows the Fourier transform infrared (FTIR) spectra of AWNP 600, AWNP 700 and AWNP
800. The IR spectra contain the characteristic bands of celluloses. In the range of stretching vibrations of $\mathrm{OH}$ groups, there is a broad band with a maximum at approximately $3400 \mathrm{~cm}^{-1}$. The band at approximately $2900 \mathrm{~cm}^{-1}$ is also strong. In the range of $900 \mathrm{~cm}^{-1}$ to $1500 \mathrm{~cm}^{-1}$, there are absorption bands forming a complex pattern with several maxima. All the samples contain residual lignin and hemicelluloses. The presence of these components is suggested by the corresponding absorption bands. Hemicelluloses can be revealed from the band at $1730 \mathrm{~cm}^{-1}$ (stretching vibrations of the $\mathrm{C}=\mathrm{O}$ groups in ester fragments) [19] and lignin, from the bands at $1595 \mathrm{~cm}^{-1}$ to $1500 \mathrm{~cm}^{-1}$ corresponding to the skeleton vibrations of the benzene ring and characteristic band at $1460 \mathrm{~cm}^{-1}$ for the bending modes of the $\mathrm{CH}$ groups [20]. These observations show that in the resulting samples almost all hydroxyls are involved in hydrogen bond, which is suggested by the shape of the absorption band in the range $3000 \mathrm{~cm}^{-1}$ to $3700 \mathrm{~cm}^{-1}$.

The maximum of the absorption band of stretching modes of $\mathrm{OH}$ groups in the spectra is practically unchanged. The WNP samples have the highest symmetry index, i.e. the sample is characterized by a fairly loose network of hydrogen bonds. The ordering index (analog of the crystallinity index in X-ray diffractometry) is determined from the ratio of optical densities at $1375 \mathrm{~cm}^{-1}$ (bending modes of $\mathrm{CH}$ groups) and $2900 \mathrm{~cm}^{-1}$. For powder materials, it is higher than that for unbleached cellulose. This means that in hydrolysis, the fraction of the amorphous part decreases and the fraction of crystalline part increases. The content of crystalline and amorphous parts in the sample can also be judged from the ratio of the intensities of the band at $1430 \mathrm{~cm}^{-1}$ (bending modes of $\mathrm{CH}$ groups) that is related to the crystalline areas, and the band at $900 \mathrm{~cm}^{-1}$ that is related to the amorphous areas $(\mathrm{C}-\mathrm{O}$ stretching modes in amorphous area).

\subsection{High resolution scanning electron mi- croscopy (HR-SEM) studies}

Fig. 3 shows the SEM images of the precarbonized and activated carbon prepared from WNP 


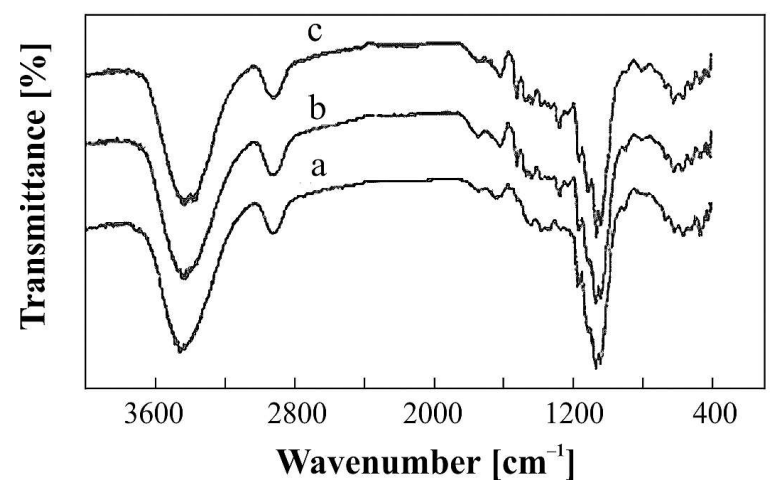

Fig. 2. FT-IR spectra of (a) AWNP 600 (b) AWNP 700 and (c) AWNP 800 carbons.

carbon. Fig. 3a shows the SEM image of PCC, which has no pores and confirms that kaolinite, talc, and cellulose components present in WNP were converted to the carbon structures [21]. The image also reveals that only carbonization has taken place. It is clear that due to some partial defects, large amount of silica has been etched out during the carbonization process. The sample AWNP 800 showed better results than AWNP 600 and AWNP 700.

Fig. 3b shows the SEM image of AWNP 600 carbon. In this image, porous structures with many cavities are observed. The cavities in AWNP 600 may be attributed to the evaporation of the activating agent, which leaves the space during the carbonization as well as the subsequent removal of untreated $\mathrm{H}_{3} \mathrm{PO}_{4}$ by washing with water [22]. Fig. 3c shows the formation of porous structure, whereas hierarchical pores can be clearly identified from Fig. 3d. It clearly shows that the hierarchical pores are more compact, uniformly distributed, and there is a good network of interconnected pores in the mesoporous range.

AWNP 800 has a well-developed interconnected hierarchical pore system containing both micropores and mesopores. The macropores can act as a reservoir for electrolyte solution buffering; whereas the mesopores and micropores are used for diffusion and adsorption of the electrolyte ions, and they are beneficial for mass transportation [23]. This kind of hierarchical porous structure can remarkably assist the fast penetration of the electrolyte into the AWNP pores, which could produce good electrochemical performance. As a result of an increase in temperature, new pores are created. In addition, there is a stable increase in both the surface area and the pore volume [24]. The SEM examinations, thus reveal that the cellulose structure decreases with increasing the firing temperature.

\subsection{Nitrogen adsorption-desorption stud- ies}

PCC, AWNP 600, AWNP 700 and AWNP 800 were characterized with respect to their surface properties: pore volume, surface area and the extent of micro and mesoporosity using $\mathrm{N}_{2}$ adsorption isotherm parameters.

Fig. 4 shows the adsorption-desorption isotherms obtained for these carbons. According to the IUPAC classification, the nitrogen adsorptiondesorption isotherms of both the samples exhibit the characteristics of type I isotherms [25]. At relatively low pressure, the adsorbed volume of nitrogen increased steeply, suggesting the existence of large amount of micropores. The broadening of the knee in the low-pressure range is attributable to mesoporosity, where PCC contains the least and AWNP 800 the most mesopores, implying that the number of micropores has increased after $\mathrm{H}_{3} \mathrm{PO}_{4}$ activation. At relatively high pressures, there is no hysteresis loop on the isotherms of PCC and AWNP, which indicates that a small number of mesopores have developed [26].

PCC contains a level of porosity in the range of $2 \mathrm{~nm}$ to $3 \mathrm{~nm}$. AWNP 800 sample consists of wider pores distributed in two well-defined pore systems: micropores and small mesopores $(2 \mathrm{~nm}$ to $8 \mathrm{~nm}$ ). This can be attributed to the combination of its highly defective structure with a large fraction of the carbon being amorphous and hence, less densely arranged than graphene. Another important aspect of activated carbons is their pore size distribution. The pore size distribution calculated by the Barrett, Joyner and Hallenda (BJH) method, indicates that the porous texture of this material is completely dominated by micro and mesopores. 


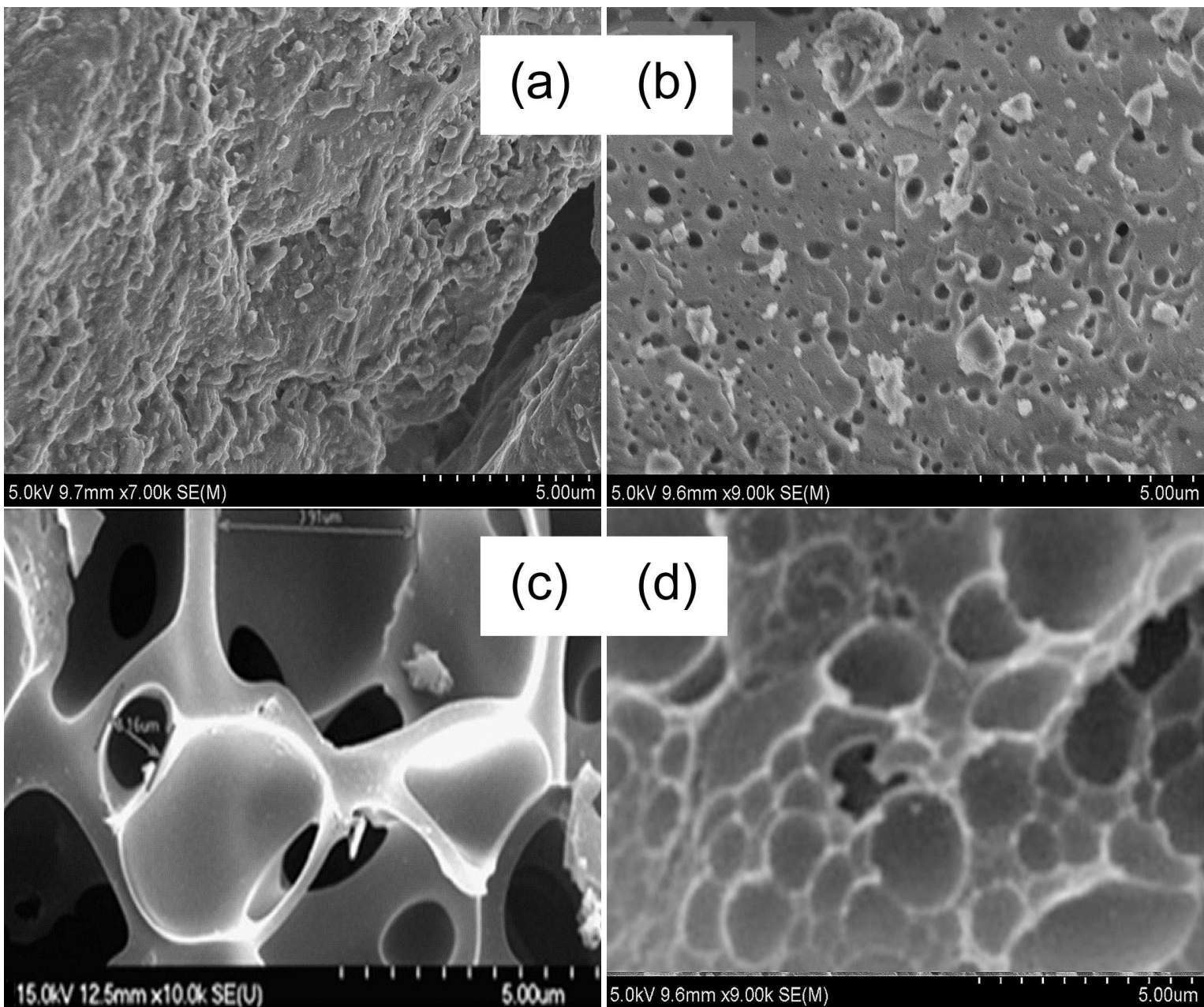

Fig. 3. Scanning electron micrographs of (a) PCC, (b) AWNP 600, (C) AWNP 700, (d) AWNP 800.

The textural parameters of the synthesized carbons are presented in Table 1. These results show that the carbon nanosheets exhibit high BET surface area and large pore volume. The pore volume increases steadily with temperature from $0.59 \mathrm{~cm}^{3} / \mathrm{g}$ (PCC) to $1.30 \mathrm{~cm}^{3} / \mathrm{g}$ (AWNP 800 ), whereas the BET surface area increases from $336.06 \mathrm{~m}^{2} / \mathrm{g}$ to $1104.08 \mathrm{~m}^{2} / \mathrm{g}$. Moreover, the analysis of the porosity by means of the Horvath-Kawazoe (H-K) method confirms that these samples are essentially microporous with the micropore volume constituting more than $80 \%$ of the total pore volume. It can be predicted that the waste paper carbon will have a perspective application in supercapacitors, and the AWNP 800 carbon will exhibit the best supercapacitive behavior among all the prepared carbon samples.
For AWNP 800, meso- and microporous surface areas are comparable. It is clear that AWNP 800 exhibits a higher specific surface area and larger average pore size, as well as a larger pore volume than AWNP 700, AWNP 600 and PCC. The reason for this is that the addition of the activating agent might cause a partial structural collapse and dissolution of the inorganic impurities, which are adverse for reducing the surface area and dissolving the non-carbons at lower temperatures. These observations make clear that the activating agents at lower temperatures may contribute to pore drilling as well as pore widening effects, whereas at higher temperatures, the pore widening effects predominate the pore drilling forces [18]. In addition, the pore structures of the activated carbon could be affected by the addition of $\mathrm{H}_{3} \mathrm{PO}_{4}$. When $\mathrm{H}_{3} \mathrm{PO}_{4}$ is 
Table 1. Sample code, surface area, pore volume, average pore diameter, and production yield of waste newspaper derived carbon.

\begin{tabular}{|c|c|c|c|c|c|c|c|c|c|c|}
\hline Sample & $\begin{array}{c}\mathrm{S}_{\mathrm{BET}}^{\mathrm{a}} \\
{\left[\mathrm{m}^{2} / \mathrm{g}\right]}\end{array}$ & $\begin{array}{c}\mathrm{S}_{\mathrm{mic}}^{\mathrm{b}} \\
{\left[\mathrm{m}^{2} / \mathrm{g}\right]}\end{array}$ & $\begin{array}{l}\mathrm{S}_{\text {meso }}^{\mathrm{c}} \\
{\left[\mathrm{m}^{2} / \mathrm{g}\right]}\end{array}$ & $\begin{array}{c}\mathrm{V}_{\text {Total }}^{\mathrm{d}} \\
{\left[\mathrm{cm}^{3} / \mathrm{g}\right]}\end{array}$ & $\begin{array}{c}\mathrm{V}_{\mathrm{Micro}}^{\mathrm{e}} \\
{\left[\mathrm{cm}^{3} / \mathrm{g}\right]}\end{array}$ & $\begin{array}{c}\mathrm{V}_{\text {Meso }}^{\mathrm{f}} \\
{\left[\mathrm{cm}^{3} / \mathrm{g}\right]}\end{array}$ & $\begin{array}{c}\mathrm{V}_{\text {Micro }} / \mathrm{V}_{\text {Total }} \\
{[\%]}\end{array}$ & $\begin{array}{c}\mathrm{V}_{\text {Meso }} / \mathrm{V}_{\text {Total }} \\
{[\%]}\end{array}$ & $\begin{array}{c}\mathrm{D}_{\mathrm{p}}^{\mathrm{g}} \\
{[\mathrm{nm}]}\end{array}$ & $\begin{array}{c}\text { Production yield } \\
\text { of carbon [\%] }\end{array}$ \\
\hline & 333.06 & 231.01 & 105.03 & 0.59 & 0.54 & 0.05 & 91.5 & 0.85 & 7.9 & 63.00 \\
\hline AWNP 600 & 621.25 & 371.39 & 250.07 & 0.96 & 0.77 & 0.19 & 80.2 & 19.7 & 7.8 & 55.66 \\
\hline AWNP 700 & 832.37 & 493.56 & 339.60 & 1.11 & 0.84 & 0.27 & 75.6 & 24.3 & 6.9 & 49.79 \\
\hline AWNP 800 & 1104.8 & 563.71 & 503.39 & 1.30 & 0.82 & 0.48 & 63.0 & 36.9 & 4.8 & 45.89 \\
\hline
\end{tabular}

${ }^{\mathrm{a}}$ BET surface area; ${ }^{\mathrm{b}}$ Micropore surface area; ${ }^{\mathrm{c}}$ Mesopore surface area; ${ }^{\mathrm{d}}$ Total pore volume; ${ }^{\mathrm{e}}$ Micropore volume; ${ }^{\mathrm{f}}$ Mesopore volume; ${ }^{\mathrm{g}}$ Average pore diameter

added, the microstructure of PCC and activated carbon will collapse, and as a result, the quantity of mesopores would increase.

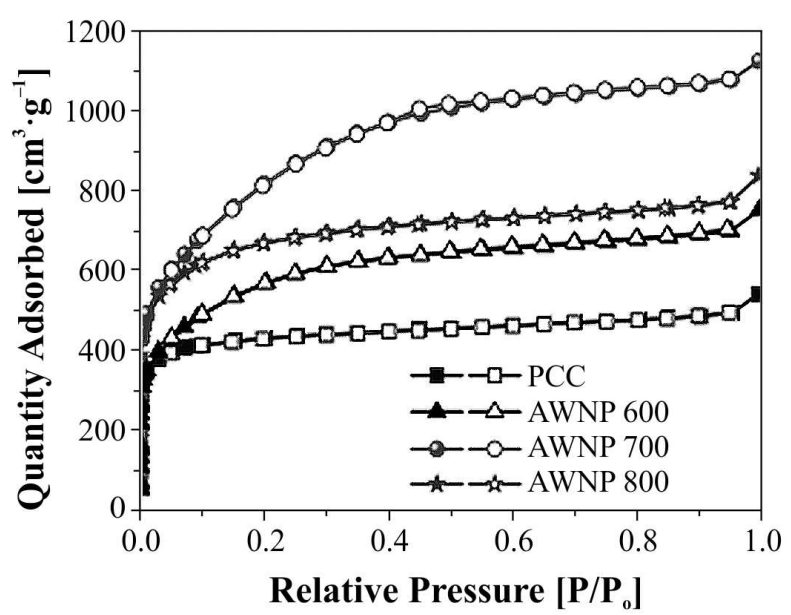

Fig. 4. Adsorption-desorption isotherms of activated carbons PCC, AWNP 600, AWNP 700, AWNP 800 carbons.

The hierarchical pore structures of the samples can facilitate diffusion of ions and improve charge accumulation. It can be predicted that the AWNP will have a perspective application in supercapacitors and the AWNP 800 will exhibit the best supercapacitive behavior among all prepared samples. This indicates that hierarchical pores created in the activated carbon samples are good for supercapacitor application. When the pore size is uniform, the hierarchical pores make maximum contribution to the EDLC due to high electroadsorption originated from the strong interaction of ions with the pore inwall [27].

\subsection{Electrical conductivity studies}

Electrical conductivity is a measure of material ability to accommodate the transport of electric charge. It is also a property that is used to describe the capability of materials to allow the electrons to flow. Electrical conduction is the movement of electrically charged particles through a transmission medium. The movement can cause an electric current in response to an electric field. Fig. 5 shows the variation of electrical conductivity $(\sigma)$ of AWNP 600, AWNP 700 and AWNP 800 samples. The ohmic conduction at room temperature for the activated carbon samples, AWNP 600, AWNP 700 and AWNP 800 at $9.63 \mathrm{kPa}$ are $9.44 \times 10^{-11}$, $2.20 \times 10^{-6}$ and $2.83 \times 10^{-3} \Omega^{-1} \cdot \mathrm{cm}^{-1}$, respectively. Similarly, the ohmic conduction at room temperature for the activated carbon samples, AWNP 600, AWNP 700 and AWNP 800 at $750.13 \mathrm{kPa}$ are $3.15 \times 10^{-10}, 1.10 \times 10^{-6}$ and $5.63 \times 10^{-2} \Omega^{-1} \cdot \mathrm{cm}^{-1}$, respectively.

It is clear from Fig. 5 that the electrical conductivity at $9.63 \mathrm{kPa}$ is low, signifying that the applied pressure is not adequate to squeeze the pores or the air gap between the carbon particles. Hence, inter-particle electronic conduction becomes more complicated. However, the electrical conductivity is increased by three-fold at $750.12 \mathrm{kPa}$. This is due to the free movement of electrons from one particle to another facilitated by the reduction of the air gap or porosity of the carbon by means of applying high pressure of compression [28, 29]. In all the cases, the value of $\sigma$ increases almost linearly with the compression exerted by the loadings. Higher 
density of carbon samples leads to higher electrical conductivity, because there is a chance for the electrons to move across them [21]. Thus, the carbon samples, AWNP 600, AWNP 700 and AWNP 800 exhibit an increase in electrical conductivity with an increase in the activation temperature.

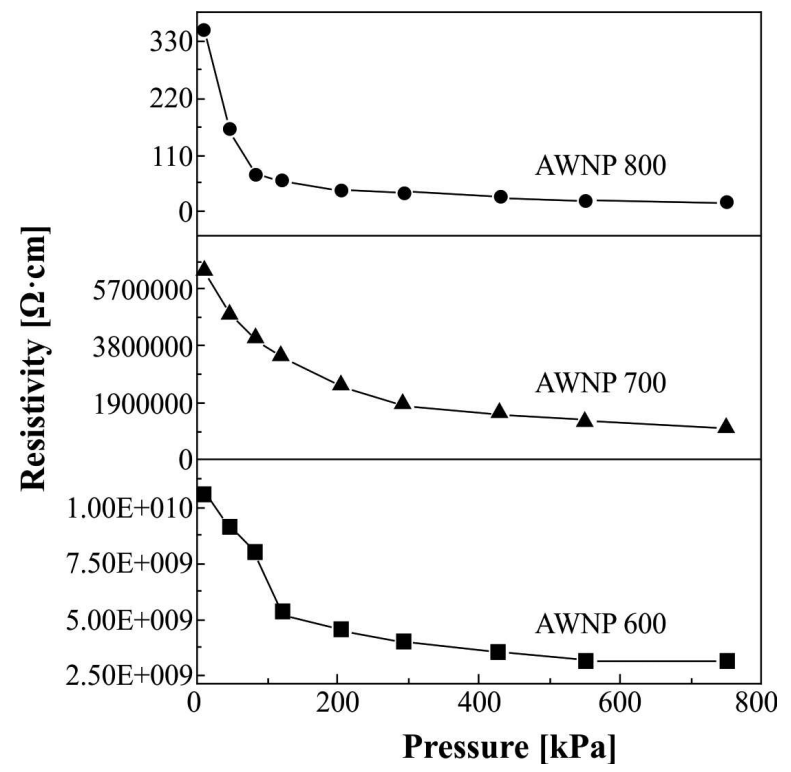

Fig. 5. Effect of compression pressure treatment on the electrical conductivity of the activated carbon samples.

According to the contact theory by Mrozowski and Holm [30, 31], $\sigma$ depends on a distance between the particles and on their average size [32, 33]. The three carbon samples (AWNP 600, AWNP 700 and AWNP 800) are constituted by the particles, which can fuse together to form aggregates. When the pressure is applied, the aggregates are forced to be in a more dense packed arrangement, creating closer contacts with neighboring aggregates. Even though the pressure plays an important effect on $\sigma$, it is not the only factor that determines its value. The morphology of the particles has also a great effect on the electrical conductivity $[34,35]$. In case of AWNP 600, the particles are irregular in shape and larger in comparison with the spherical particles of AWNP 700 and AWNP 800 as shown in Fig. 3.

\subsection{Evaluation of electrochemical prop- erties}

The electrochemical performances of the devices were evaluated at room temperature. Fig. 6 exhibits the typical cyclic voltammograms (CV) of AWNP 600, AWNP 700 and AWNP 800 using $1 \mathrm{M}$ $\mathrm{H}_{2} \mathrm{SO}_{4}$ as an electrolyte at the scan rate of $2 \mathrm{mV} / \mathrm{s}$ to $3 \mathrm{mV} / \mathrm{s}$. The effect of AWNP carbon was investigated in order to elucidate the charge storage mechanism. Clearly, the CV curves obtained at different temperatures are close to quasi-rectangular shape. The shape indicates an excellent capacitive behavior, low contact resistance, and no faradic processes. This character implies that the electrode material is efficiently used for charge storage; the electrolyte has easy access to electrodes which results in better ion diffusion. The increase in the area of the AWNP curves indicates an enhancement of the specific capacitance.

The total capacitance of the prepared WNP carbons was calculated directly from the currentvoltage curves as given below:

$$
C=\left(\frac{I_{a}-I_{c}}{2 v \cdot m}\right)=\left(\frac{\Delta I}{2 v \cdot m}\right)
$$

where $\mathrm{C}$ is specific capacitance $(\mathrm{F} / \mathrm{g})$, $\mathrm{v}$ is potential scan rate $(\mathrm{V} / \mathrm{s}), \mathrm{m}$ is mass of the WNP carbon powder, $I_{a}$ and $I_{c}$ are the average anodic and cathodic currents, respectively. To determine the capacitance as a function of potential, $\Delta \mathrm{I}$ values were obtained at various potentials. The specific capacitance of the WNP carbon electrodes is in the range of $276 \mathrm{~F} / \mathrm{g}$ to $380 \mathrm{~F} / \mathrm{g}$ at a scan rate of $2 \mathrm{mV} / \mathrm{s}$, i.e. AWNP 600: $276 \mathrm{~F} / \mathrm{g}$, AWNP 700: 334F/g and AWNP 800: $380 \mathrm{~F} / \mathrm{g}$. Therefore, the galvanostatic charge-discharge behavior has been studied for AWNP 800 alone.

Fig. 7 shows the CV results of the selected sample AWNP 800, which has the best electrochemical performance at various scan rates. At $10 \mathrm{mV} / \mathrm{s}, 20 \mathrm{mV} / \mathrm{s}, 30 \mathrm{mV} / \mathrm{s}, 50 \mathrm{mV} / \mathrm{s}$ and $100 \mathrm{mV} / \mathrm{s}$, the curves present rectangular shapes for the charge/discharge processes. At the scan rate of $100 \mathrm{mV} / \mathrm{s}$, a rectangular shape curve with only a slight deviation from the ideal rectangular shape can be observed, which indicates a pure EDLC 

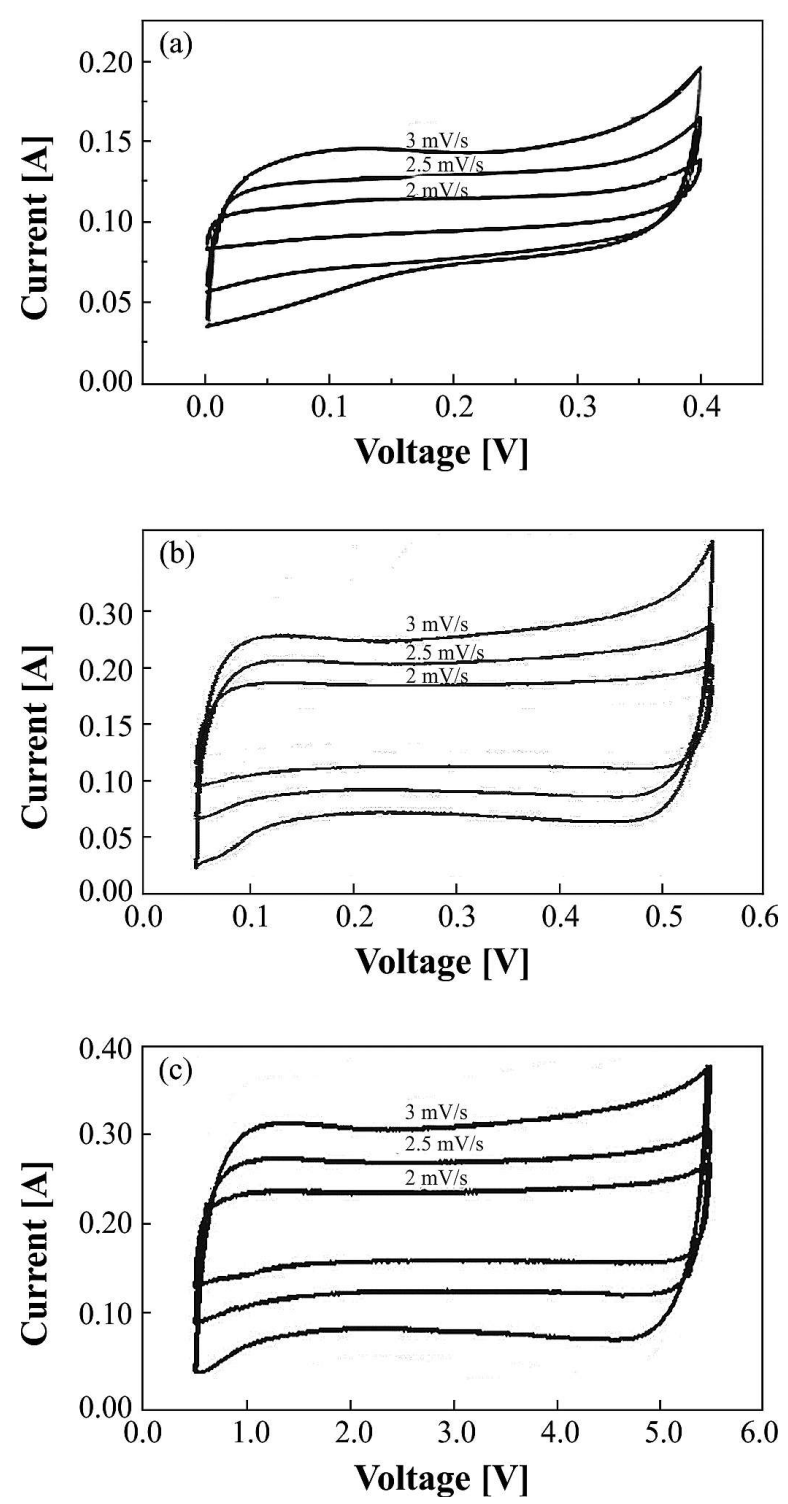

Fig. 6. Cyclic voltammograms of (a) AWNP 600, (b) AWNP 700, (c) AWNP 800 using $1 \mathrm{M} \mathrm{H}_{2} \mathrm{SO}_{4}$ as electrolyte at different scan rates.

behavior and rapid formation of a double-layer even at high rates. This result suggests a good match between the pore size and electrolyte ions size. On the other hand, it can be observed that even at a scan rate as high as $100 \mathrm{mV} / \mathrm{s}$, it retains about $85 \%$ of their specific capacitance.

Electrochemical impedance spectroscopy is a potent means to study the capacitive behavior of waste paper carbon materials. Fig. 8 displays

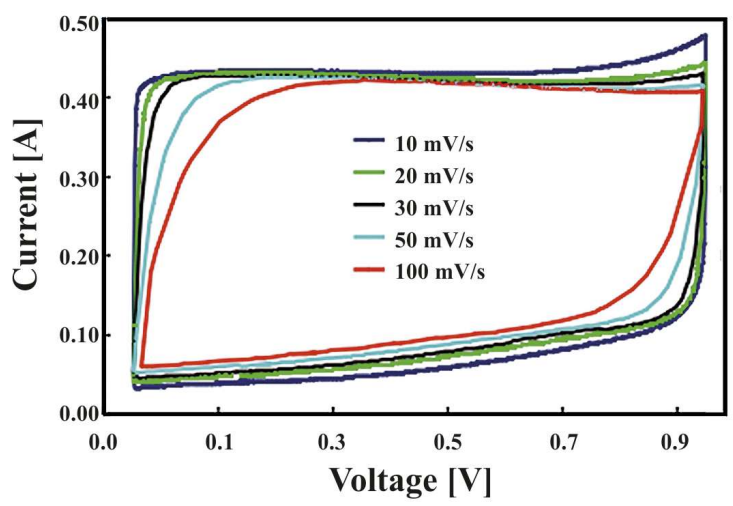

Fig. 7. Cyclic voltammogram of AWNP 800 at various scan rates ranging from $10 \mathrm{mV} / \mathrm{s}$ to $100 \mathrm{mV} / \mathrm{s}$.

Nyquist plots of impedance spectra measured for AWNP 800 electrode. The Nyquist plot for AWNP 800 has the impedance curve going nearly parallel to $-Z$ " axis, which is close to the case of an ideal capacitor. The $\mathrm{x}$-intercept of the Nyquist plot provides the total series resistance, which plays an important role at higher charge-discharge rates. This depends on the intrinsic resistance of the electrode material, current collector, and the electrolyte. This behavior can be attributed to the unique structural properties of the AWNP 800, which combine a thin morphology (short ion diffusion paths) and a bimodal microporosity with interconnected narrow and wide micropores that improve the ion diffusion rates.

The total series resistance observed for AWNP 800 was $\sim 1.1 \Omega$. The inset of Fig. 8 shows the high frequency part of the Nyquist plot. A total ESR of $1.3 \Omega$ was obtained for the device, which included the total series resistance as well as the charge transfer resistance. At lower frequencies, a straight sloping line represents diffusive resistance (Warburg impedance) of the electrolyte in the pores of the host carbon. AWNP 800 showed higher angles than $45^{\circ}$, indicating the suitability as the electrode for EDLCs, due to its effective mesopores, which facilitate the transport of electrolyte ions.

Fig. 9 illustrates the charge-discharge behavior of AWNP 800. It shows a symmetric triangular shape illustrating that the AWNP 800 sample has a typical supercapacitive behavior of porous 


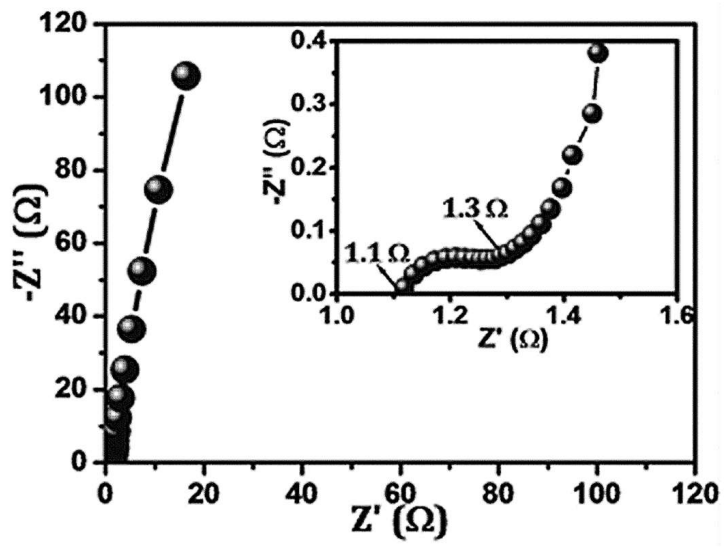

Fig. 8. Nyquist plot of the as-prepared AWNP 800.

carbon and excellent capacitive reversibility, which is in good agreement with the CV observations. This proves that the interconnected porous structure of the carbon material helps in the diffusion of electrolyte ions even in the gel form and hence, the material is able to deliver equal performance for liquid electrolyte. The micropores act as active sites for the double layer formation, whereas the mesopores and macropores act as the channels for the penetration of the electrolyte into the inner layers of the electrode material. In the case of AWNP 800 , the pore density is maximum in the pore size range of $1 \mathrm{~nm}$ to $3 \mathrm{~nm}$. Since this pore size matches fairly well with the size of the solvated ions, a high specific capacitance is expected, as observed.

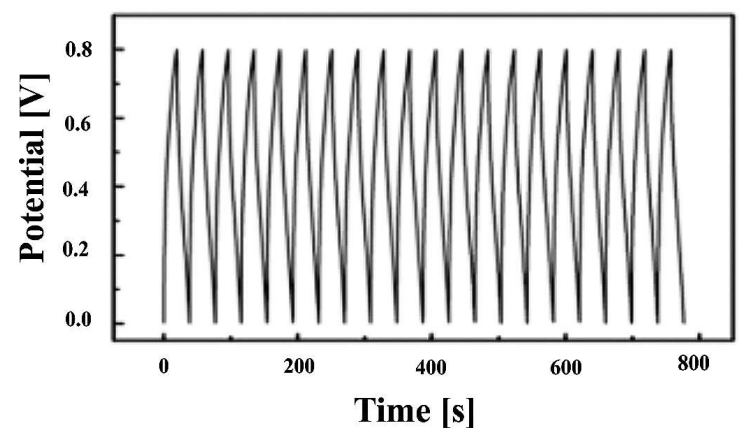

Fig. 9. Charge-discharge curves of AWNP 800 at fixed $2 \mathrm{Ag}^{-1}$.

Fig. 10 shows the cyclic stability of AWNP 800. It can be seen that the specific capacitance gradually decreases with an increase of the cycle number and $85 \%$ of the initial specific capacitance remains after 5000 cycles. The decrease in the capacitance can be due to the reaction between the electrolyte and the electrode. The results reveal the excellent stability of the waste paper based carbon electrodes for high-performance EDLCs. All the electrochemical tests demonstrate that the as-fabricated waste paper based carbon electrodes have a superior supercapacitive performance and are promising for use in EDLCs.

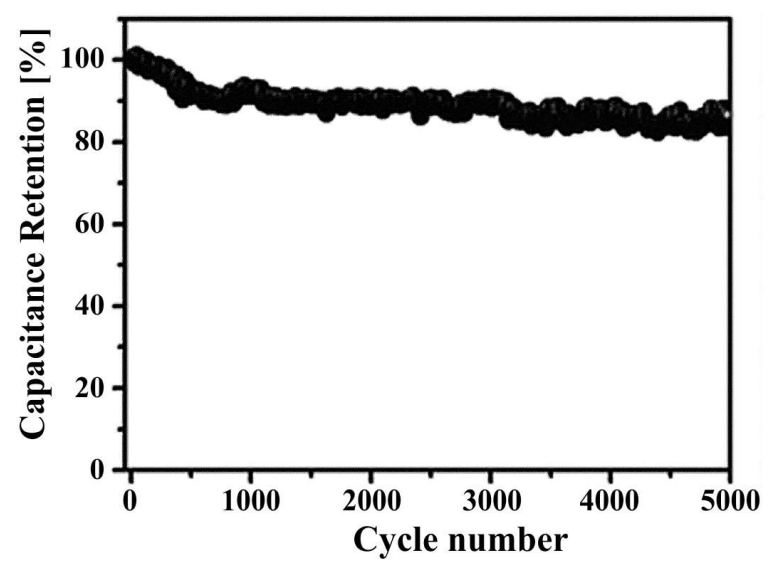

Fig. 10. Cycling stability of AWNP 800 electrode.

\subsection{Device fabrication of AWNP 800}

A polyvinyl alcohol (PVA) and phosphoric acid $\left(\mathrm{H}_{3} \mathrm{PO}_{4}\right)$ polymer gel electrolyte was prepared as described in the literature reports and functioned as both the electrolyte and separator [24]. Usually, in industrially produced supercapacitors, aqueous solution of alkali (decomposition voltage of $1.23 \mathrm{~V}$ and electrical conductivity of $1.0 \mathrm{~S} / \mathrm{cm}$ ) or organic electrolytes (decomposition voltage of $2.7 \mathrm{~V}$ and electrical conductivity of $0.01 \mathrm{~S} / \mathrm{cm}$ ) are used. In our study, the inner electrode consisted of $\sim 2 \mathrm{~cm}$ AWNP 800 , which was coated with silver paint to create a region for electrical connection. Prior to coating with the polymer electrolyte, a pre-charging procedure was performed on the inner electrode. While, sub-merged in $1 \mathrm{M} \mathrm{H}_{3} \mathrm{PO}_{4}$ electrolyte, a $0.7 \mathrm{~V}$ (vs. SCE) constant potential was applied for $500 \mathrm{~s}$, after which the electrode was immediately removed from the electrolyte. Zakhidov et al. [36] showed that high double-layer 
charge densities can be retained after removing the sample from their electrolytes.

The pre-charged electrode was then coated with the $\mathrm{PVA} / \mathrm{H}_{2} \mathrm{O} / \mathrm{H}_{3} \mathrm{PO}_{4}$ solution by several iterations of dip coating and re-twisting, which simultaneously removed the excess gel from the strands and compacted the structure. After drying overnight in open air, a uniform solid polymer coating was obtained on the electrode. The outer electrode was then applied by wrapping thin AWNP 800 around the coated inner electrode. The total mass of the outer electrode sheets was matched to the inner electrode mass for each device. The sheets were wrapped around the coated inner electrode, starting $\sim 2 \mathrm{~cm}$ from one end and extending past to the opposite end of the electrode. This excess was twisted upon itself that extended several centimeters past the end of the inner electrode. Silver paint was applied to the end $(\sim 2 \mathrm{~cm})$.

The final step of the fabrication processes consisted of a second application of the polymer electrolyte solution to coat the outer electrode. Careful attention was paid to leave the silver coated regions at the ends of each electrode free from any contact with electrolytes or polymers. The active region of each device (region in which the two electrodes overlapped one another) was $\sim 50 \mathrm{~mm}$ in length and $\sim 500 \mu \mathrm{m}$ in diameter. Fig. 11 and Fig. 12 show the size, dimensions and schematic representation of the supercapacitor electrode cell. Table 2 shows the characteristics of lab made supercapacitor.

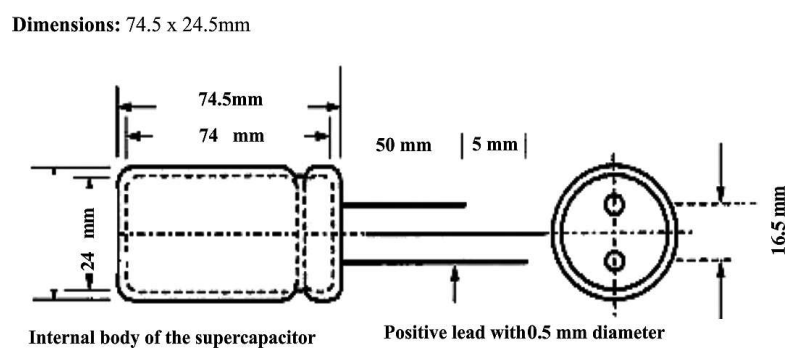

Fig. 11. Size and dimensions of the supercapacitor electrode.

Details of fabricated supercondutor are given in Fig. 11.

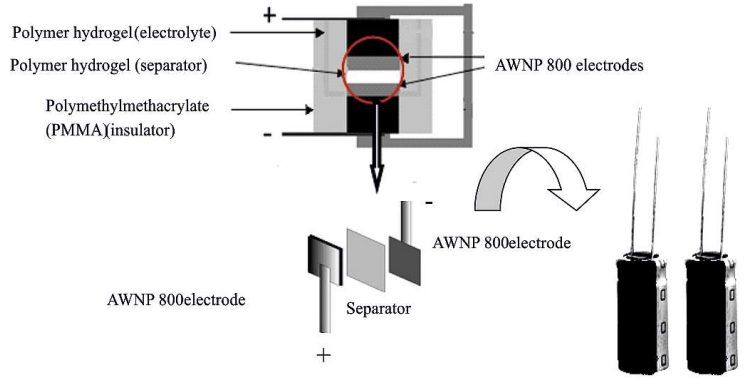

Fig. 12. Schematic representation of AWNP 800 supercapacitor electrode cell.

\subsection{Production yield}

Table 1 shows the production yield of the asprepared WNP carbon. The yield of PCC was around $63 \%$. However, after the acid activation and washing, the carbon yields corresponding to samples AWNP 600, AWNP 700, and AWNP 800 were in the range of $55.6 \%$ to $45.8 \%$. The difference in the carbon yield was mainly due to the removal of volatile substances through the openings of the closed pores at higher temperatures. Putun et al. [34] reported that the increased temperature led to a decreased yield of carbon. When the temperature was raised, there was a rise in ash and in fixed carbon percentage and there was a decrease in the volatile matter. Therefore, higher temperature yielded charcoals of greater quality. The decrease in the yield with increasing temperature was due to the greater primary decomposition of biomass at higher temperatures or through the secondary decomposition of the char residue. As the temperature increased, vapors inside the cracked particle had to stay there for a shorter time, and hence the yield was decreased [35].

\section{Conclusions}

In summary, we have developed a simple method to produce a novel class of waste newspaper based carbon electrodes for fabricating highperformance supercapacitors with environmentally friendly approach (Table 2). We found that AWNP 800 provided better capacitance than AWNP 700, AWNP 600 and PCC. XRD analysis confirmed the formation of graphitic carbon structure and 
Table 2. Characteristics of lab made supercapacitor.

\begin{tabular}{lc}
\hline \multicolumn{1}{c}{ Characteristics } & Lab made supercapacitor \\
\hline \hline Operating temperature range & $-35^{\circ} \mathrm{C}$ to $+70{ }^{\circ} \mathrm{C}$ \\
Capacitance & $100 \mathrm{~F}$ to $380 \mathrm{~F}$ \\
Rated voltage & $1200 \mathrm{~V}$ to $3000 \mathrm{~V}(\mathrm{DC}$ current) Protection against overvoltage \\
Tolerance & $\pm 10 \%$ to $\pm 15 \%$ \\
Dissipation factor & $\leqslant 0.5 \times 10^{-3}$ (measured at $100 \mathrm{~Hz}$ and $25{ }^{\circ} \mathrm{C}$ ) \\
Life expectancy & 10,000 hours at $85^{\circ} \mathrm{C}$ (life span of at least 20 years) \\
Environment friendly & Green product \\
\hline
\end{tabular}

lower crystallinity in the activated carbon structure. The use of $\mathrm{H}_{3} \mathrm{PO}_{4}$ during the inclusion process prior to the chemical activation made the process simpler and cheaper. It was found that the chemical activation at $800{ }^{\circ} \mathrm{C}$ was optimum to produce a highly porous carbon, and it was confirmed by SEM analysis. The conductivity and its variation under compression pressure were directly related to the density of the carbons. These results clearly indicate that the prepared material is excellent for supercapacitor performance with WNP as an electrode material for energy storage devices. These properties possibly enable these cellulosic wastes to act as a source of carbonaceous materials for the development of high-performance supercapacitors.

\section{References}

[1] Chen X., Li C., Gratzel M., Kostecki R., MaO S.S., Chem. Soc. Rev., 41 (2012), 7909.

[2] Sanada Y., Suzuki M., Fujimoto K., Activated Carbon: Its fundamentals and applications, Kodan-sha, Tokyo, 1975.

[3] PRZybysz K., Paper technology, Part 2, WSiP, Warszawa, 1997.

[4] Biedermann M., Grob K., Eur. Food. Res. Technol., 230 (2010), 785.

[5] Shin H J., Kim C.J., Kiм S.B., Biotechnol. Bioprocess. Eng., 14 (2009), 349.

[6] JAPAN PAPER MANUFACTURING FEDERATION, Paper and Pulp (in Japanese), 564 (2014), 13.

[7] Murphy R.J., Silkebakkan D., Chatchupong T., Florida centre of solid and hazardous waste management, University of Florida, Florida, 1990.

[8] Wyman C.E., DAle B.E., Elander R.T., Bioresour. Technol., 96 (2005), 1959.

[9] Lu W., Hartman R., Qu R.L., J. Phys. Chem. Lett., 2 (2011), 655.

[10] DekKer M., Cooney D O., Activated Charcoal: Antidotal and other medical uses, New York, 1980.
[11] BAKer F.S., Miller C.E., RePIK A.J., Encyclopedia of chemical technology, John Wiley and Sons, New York, 1992.

[12] Kalpana D., Cho S.H., LeE S.B., J. Power Sources, 190 (2009), 587.

[13] Rodriguez-Reinoso F., Pastor A.C., Marsh H., Carbon, 38 (2000), 379.

[14] Wang T.H., Tan S.X., Liang C.H., Carbon, 47 (2009), 1867.

[15] PARAKNOWITSCH J.P., Zhang J., SU D., Thomas A., Antonietti M., Adv. Mater., 22 (2010), 87.

[16] InAGaki M., New Carbon Mater., 24 (2009), 193.

[17] Zhang G. Q., Zhang S.T., J. Solid State Electrochem., 13 (2009), 887.

[18] Viswanathan B., Neel P.I., Varadarajan T.K., Methods of Activation and Specific Applications of Carbon Materials, National Centre for Catalysis Research, Indian Institute of Technology Madras, Chennai, 2009.

[19] Bazarnova N.G., Karpova E.V., KatraKov I.B., Methods of Study of Wood and its Derivatives, Altai State University, Barnaul, 2002.

[20] Mironov V.A., YanKovskiI S.A., Spectroscopy in organic chemistry, Khimiya, Moscow, 1985.

[21] AdinaVEen T., Kennedy L.J., ViJaya J.J., J. Indian Eng. Chem., 19 (2013), 1470.

[22] Senthilkumar S.T., Senthilkumar B., BaLAJI S., Mater. Res. Bull., 46 (2011), 413.

[23] Fang B Z., KIM J.H., KIM M.S., Acc. Chem. Res., 46 (2013), 1397.

[24] Subramanian V., Luo C., Stephan A.M., J. Phys. Chem. C, 111 (2000), 7527.

[25] LiU X., LiU W., Zhang Z., Mater. Lett., 116 (2014), 304.

[26] Zhang P., Cui X., Chen J., Liu J., Solid State Electrochem., 6 (2013), 1749.

[27] Yamada H., Nakamura H., Nakahara F., Moriguchi I., Kudo T., J. Phys. Chem. C, 111 (2007), 227.

[28] Mrozowski S., Studies of carbon powders under compression, in: Proceedings $3^{\text {rd }}$ Carbon Conference in Buffalo, Pergamon Press, 1959, p. 495.

[29] HoLm R., Electrical Contacts, Almquist and Wiksells, Stockholm, 1946, p. 214. 
[30] Holm R., Electric Contacts: Theory and Application, Reprint of the $4^{\text {th }}$ completely rewritten ed., Springer Verlag, Berlin/Heidelberg, 1967, p. 135.

[31] Kinoshita K., Carbon: Electrochemical and Physicochemical Properties, New York, John Wiley and Sons Ltd., 1988.

[32] Pantea D., Darmstadt H., Kaliaguine S., Carbon, 39 (2001), 1147.

[33] Donnet J.B., Voet A., Carbon Black: Physics, Chemistry, and Elastomer Reinforcement, Marcel Dekker, New York, 1976.
[34] Putun A.E., Ozbay N., Onal E.P., Putun E., Fuel. Process. Technol., 86 (2005), 1207.

[35] Wigman T., Carbon, 27 (1989), 13.

[36] Zakhidov A.A., Suh D.S., Kuznetsov A.A., BARISCI J.N., MUNOZ E., DALTON A.B., Collins S., Ebron V.H., Zhang M., Ferraris J.P., Zakhidov A.A., Baughman R.H., Adv. Funct. Mater., 19 (2009), 2266.

Received 2015-06-11

Accepted 2016-05-13 